\title{
Constant Temperature from Phase Space View of Ensembles of Excited States
}

\author{
LIU Shubin \\ Research Computing Center, University of North Carolina, Chapel Hill, North Carolina 27599-3420, USA. \\ Email:shubin@email.unc.edu
}

Several decades ago Ghosh, Berkowitz and Parr ${ }^{1}$ rewrote the ground-state density functional theory (DFT) into a local thermodynamics. They obtained a phase-space distribution function by maximizing the phase-space Shannon information entropy with fixed density and local kinetic energy density (of the DFT). A local Maxwell-Boltzmann distribution function was resulted. They introduced the concept of local temperature. Though, there is only an analogy between the thermodynamics and the density functional theory of a single atom or molecule, it is still amazing that these systems can be considered as an "ideal gas". Even the fundamental equations ${ }^{2}$ can be derived and the time-dependent density functional theory can be formalized as a thermodynamics with balance equations ${ }^{3}$ for the energy and entropy.

The thermodynamical transcription has been recently extended to ensembles of excited states a few years ago by Nagy ${ }^{4}$. In its original formulation density functional theory was a groundstate theory, but later was extended to excites states as well ${ }^{5,6}$. The author showed that the ensemble theory of excites states can be reformulated as a local thermodynamics and ensemble local temperature and entropy can be defined.

The Ghosh-Berkowitz-Parr theory has the peculiar property that the local temperature is not uniquely defined, which is troublesome and puzzling. It is the consequence of the ambiguity of the kinetic energy density, which might have a few different forms. In principle, any kinetic energy density that integrates to the same kinetic energy can do. In a recent paper ${ }^{7}$ Nagy proved that it is possible to select the kinetic energy density so that the local temperature be a constant for the whole system under consideration. Moreover, it turned out that, as her proof shows, the kinetic energy density corresponding to the constant temperature also maximizes the information entropy at the same time.

As a continuation of her previous studies, in the present paper (This paper is published online in the Journal of Acta Physico-
Chimica Sinica, doi: 10.3866/PKU.WHXB201709221) ${ }^{8}$, Professor Ágnes Nagy (University of Debrecen, Hungary) has extended the idea of constant temperature to ensembles of excited states. The generalization is based on the fact that the ensemble kinetic energy density is not defined uniquely either. The author has proved that the ensemble temperature corresponding to the extremum phase-space information entropy is constant. The ensemble kinetic energy density simultaneously maximizing the phase-space information entropy is found to be proportional to the ensemble density, while the ensemble temperature is proportional to the ensemble kinetic energy. Interestingly, the Maxwell-Boltzmann distribution function still holds true and the entropy has the form of the Sackur-Tetrode expression of the classical ideal gas ${ }^{8}$.

\section{References}

(1) Ghosh, S. K.; Berkowitz, M.; Parr, R. G. Proc. Natl. Acad. Sci. U. S. A. 1984, 81,8028 .

(2) Nagy, Á.; Parr, R. G. J. Mol. Struct. (Theochem) 2000, 501, 101. doi: 10.1016/S0166-1280(99)00418-2

(3) Nagy, Á. J. Mol. Struct. (Theochem) 2010, 48, 943. doi: 10.1016/j.theochem.2009.10.010

(4) Nagy, Á. Indian J. Chem. A 2014, 53, 965.

(5) Theophilou, A. K. J. Phys. C 1978, 12, 5419. doi: $110.1088 / 0022-3719 / 12 / 24 / 013$

(6) Gross, E.K.U.; Oliveira, L.N.; Kohn, W. Phys. Rev. A 1988, 37, 2805; 2809; 2821. doi: 10.1103/PhysRevA.37.2805; 10.1103/PhysRevA.37.2809; 10.1103/PhysRevA.37.2821

(7) Nagy, Á. Int. J. Quantum Chem. 2017, 117. doi: 10.1002/qua.25

(8) Nagy, Á. Acta Phys. -Chim. Sin. 2018, 34. 492. doi: 10.3866/PKU.WHXB201709221 